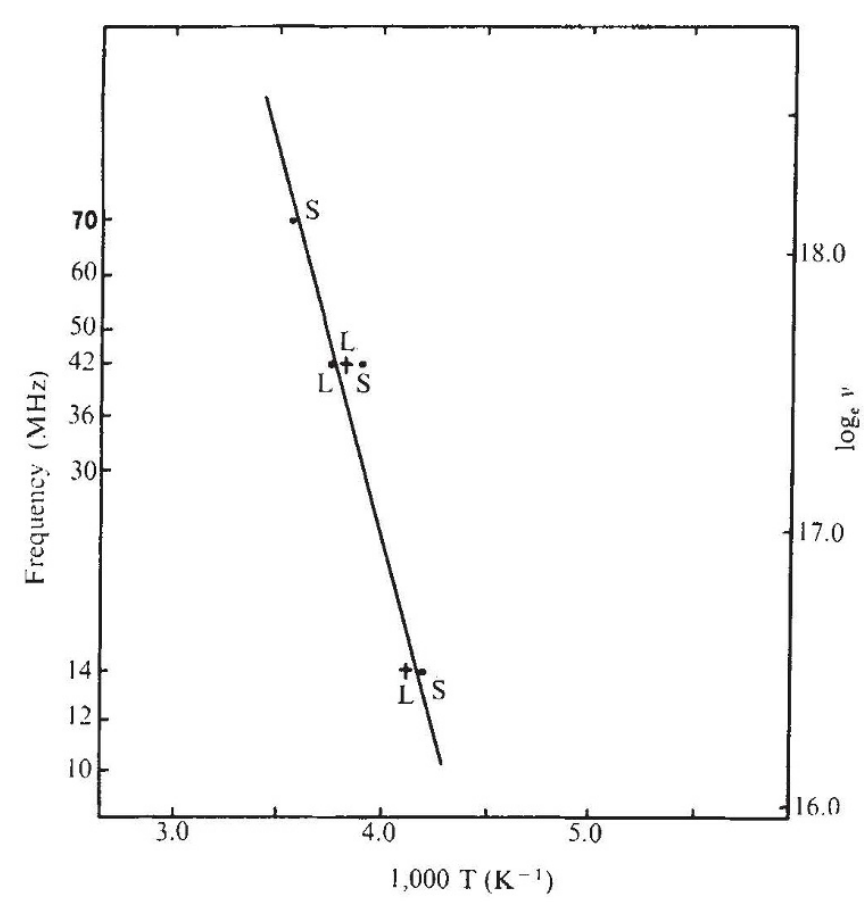

Fig. 3 Arrhenius plot to determine the activation energy and attempt frequency corresponding to the Bordoni peak, induced by passing through the transition. $\bullet$, Sample $\mathrm{A}$; +, sample B; L, longitudinal waves; S, shear waves.

variations were found to be small. The activation energy, $E$, was found to be $0.24 \pm 0.05 \mathrm{eV}$ and the attempt frequency, $v_{0}$, $1.5 \pm 0.4 \times 10^{12} \mathrm{~Hz}$. Applied to Bordoni peaks in f.c.c. metals in general this procedure gives activation energies of the order of $0.1 \mathrm{eV}$ : for copper, $E$ is $0.1-0.15 \mathrm{eV}$ (with $v_{0}=10^{10}-10^{13}$ $\mathrm{Hz}$ ); for aluminium, $E$ is $0.25 \mathrm{eV}\left(v_{0}=10^{14} \mathrm{~Hz}\right)$ (ref. 5). The peak parameters obtained for indium-thallium alloy studied here, fall into the same range. It is interesting that there is no significant difference, within the limits of experimental error, between the activation energies obtained for the longitudinal and the transverse modes. A difference can occur, however, when the resolved stress component differs on the slip plane corresponding to the polarisations of the longitudinal and transverse ultrasonic waves ${ }^{9}$.

The general features of the peak in the ultrasound attenuation in the alloy are consistent with those of Bordoni peaks. In other materials such peaks have been shown previously to result from dislocation motion, and to occur at high dislocation densities. The peaks in the 27 atom $\% \mathrm{Tl}$ alloy were formed after passage of the crystals through the martensitic transition, and back. If a dislocation damping mechanism is assumed, then not only is the frequency dependence of the peak temperature accounted for, but so is the observation that the peak appears initially only on warming. The strains involved during the transformation must produce a large number of dislocations which then interact with the ultrasound waves as the sample is warmed up, and anneal out when it is held at room temperature for several hours $(300 \mathrm{~K}$ is approximately 0.7 of the melting temperature). Therefore, subsequent cooling does not result in the reappearance of the peak. But if after several cycles, the dislocations become pinned sufficiently for short period annealing to become ineffective in causing their removal, then the peak will appear in both the cooling and the warming parts of the cycle, as is observed. The attenuation peak results provide experimental evidence of the production of dislocations during the martensitic transformation.

D. J. GUNTON

G. A. SAUNDERS

Department of Applied Physics and Electronics,

University of Durham,

South Road, Durham, UK
Received April 9, 1974.

${ }^{1}$ Guttman, L., Trans. Am. Inst. Min. Engrs., 188, 1472 (1950).

${ }^{2}$ Bowles, J. S., Barrett, C. S., and Guttman, L., Trans. Am. Inst. Min. Engrs., 188, 1478 (1950).

${ }^{3}$ Gunton, D. J., and Saunders, G. A., Solid State Commun. 14, 865 (1974).

${ }^{4}$ Bordoni, P. G., J. acoust. Soc. Am., 26, 495 (1954).

${ }^{5}$ Niblett, D. H., Physical Acoustics, Vol. IIIA, (edit. by Mason, W. P.), 77 (Ácademic Press, London, 1966).

${ }^{6}$ Pace, N. G., and Saunders, G. A., Proc. R. Soc., A326, 521 (1972).

7 Birnbaum, H. K., and Levy, M., Acta metall., 4, 84 (1956).

${ }^{8}$ Baxter, W. J., and Wilks, J., Acta metall., 11, 979 (1963).

9 Saunders, G. A., and Seddon, T., Physics Chem. Solids, 31, 2495 (1970).

\section{Determination of the gas constant by an acoustical method}

A NEW value for the gas constant, $R_{0}$, has been obtained from measurements of the velocity of sound, $c$, in argon at the temperature of the triple point of water. Velocity measurements were made using a low frequency variable-path acoustic interferometer, operating at a frequency of $5.6 \mathrm{kHz}$ (ref. 1). Ninety eight independent measurements of sound velocity were made over a range of pressures from $0.3-2.0$ atm. A quadratic least squares fit was made of $c^{2}$ against pressure, and the resulting acoustic isotherm was extrapolated to zero pressure to yield a value for $c_{0}^{2}$, the velocity in the limit of low pressures. The gas constant was then obtained from the relationship

$$
c_{0}^{2}=\gamma R_{\mathrm{o}} T / M
$$

where $\gamma=5 / 3, T=273.16 \mathrm{~K}, M=39.9478 \mathrm{~g} \mathrm{~mol}^{-1}$ and $c_{*}^{\prime \prime}$ was the experimentally determined value of $94,768.9+2.1 \mathrm{~m}^{2} \mathrm{~s}^{-2}$. The uncertainty quoted for $c_{i j}^{\prime \prime}$ is the standard error on the intercept of the acoustic isotherm.

The value for the gas constant thus obtained is

$$
R_{\mathrm{o}}=8,315.59+0.18 \mathrm{~J} \mathrm{~K}^{-1} \mathrm{kmol}^{-1}
$$

The uncertainty quoted here is the standard error in the result, which stems from the standard error in $c_{0}^{2}$ arising from random errors in the measurements of sound velocity. It is equivalent to a standard error of 22 parts per million (p.p.m.) in $R_{0}$.

The largest systematic uncertainty is that arising from the uncertainty in the isotopic composition of the argon used in the measurements, and is thought not to exceed 7 p.p.m. Errors in temperature measurement, acoustic frequency and the measurement of displacement in the interferometer are considered negligible, and errors resulting from chemical impurities in the argon are not thought to lead to a systematic uncertainty greater than 3 p.p.m. The major uncertainty in the result is thus that arising from random errors in the measurement of velocity.

Full details of the experimental method and results will be published.

This new value for $R_{\mathrm{o}}$ is greater by $1.18 \mathrm{~J} \mathrm{~K}^{-1} \mathrm{kmol}^{-\mathbf{i}}$ (142 p.p.m.) than that based upon measurements of the density of oxygen and quoted as $8,314.41 \pm 0.26 \mathrm{~J} \mathrm{~K}^{-1} \mathrm{kmol}^{-1}$ (ref. 2).

T. J. QUINN

T. R. D. Chandler

A. R. COLClOUGH

National Physical Laboratory,

Teddington, Middlesex TWII OLW, UK

Received May 14, 1974.

1 Quinn, T. J. A., Atomic masses and fundamental constants 4: proceedings of the international conference, National Physical Laboratory 1971 (edit. by Sanders, J. H., and Wapstra, A. H., 529-33 (Plenum Press, London, 1972).

2 Cohen, E. R., and Taylor, B. N., Journal of Physical and Chemical Reference Data, 2, 566 (1973). 